\title{
Transmission Electron Backscatter Diffraction (tEBSD) analysis of Au Thin Films
}

\author{
Eliot Estrine $^{1}$, Nicholas Seaton $^{2}$, Prabesh Dulal $^{1}$ and Bethanie Stadler ${ }^{1}$ \\ ${ }^{1 .}$ ECE Department, University of Minnesota, Minneapolis, MN \\ 2. Characterization Facility, University of Minnesota, Minneapolis, MN
}

Identifying and characterizing nanoscale crystallographic features is vital for understanding deformation in plasmonic devices and developing more robust plasmonic structures. Here, advanced characterization processes using transmission Electron Back Scatter Diffraction (tEBSD) were developed to analyze thin film crystal structure and its role in plasmonic device stability. The specific crystalline features of interest are film texture, grain boundary configuration and grain size. These results will hopefully contribute to the successful implementation of Heat Assisted Magnetic Recording (HAMR) as well as improve plasmonic device reliability in other applications.[1]

Nanoscale deformation has significant differences from macroscale melting. Higher energy gold $\{110\}$ facets have higher surface energy than either $\{111\}$ or $\{100\}$ facets and have been seen to reconfigure into a lower energy configuration when exposed to large temperatures or energies.[2] The presence of low energy grain boundaries, including low angle and coincidence site lattice (CSL) boundaries can also result in more robust nanostructures.[3] EBSD is a promising technique for studying such grain boundaries, but the resolution of EBSD is limited by the interaction volume of the electrons within the sample. Recently, improvements in EBSD resolution have been obtained using thin specimens or nanoparticles in a transmission configuration.[4] In transmission mode, the electron interaction volume is much smaller, and there is no scattering from the substrate. This relatively new characterization technique offers a way of directly observing morphology and failure mechanisms in films and nanostructures with small grain sizes.

For this work, sputtered $\mathrm{Au}$ films were studied by both conventional (reflection) and transmission EBSD. The Monte Carlo simulator CASINO was used to calculate electron trajectories within the sample, Figure 1. Simulations showed that a transmission configuration reduces the lateral spread of backscattered electrons to $4 \mathrm{~nm}$ (Figure 1A) compared with approximately $100 \mathrm{~nm}$ using conventional EBSD (Figure 1B).

Samples were prepared by sputtering $25 \mathrm{~nm}$ of $\mathrm{Au}$ on commercial TEM windows made of thin $\mathrm{Si}_{3} \mathrm{~N}_{4}$ membranes. Although designed for TEM imaging, these substrates also work exceptionally well for tEBSD since the thin $\mathrm{Si}_{3} \mathrm{~N}_{4}$ is almost entirely transparent to the electrons. A customized sample holder was fabricated to keep a thin sample at approximately 10 degrees off normal during imaging.

Using tEBSD, $80-90 \%$ grain indexing was achieved over a $1 \mu \mathrm{m}$ by $1 \mu \mathrm{m}$ area, Figure 2 . It is possible to increase indexing to greater than $90 \%$ by applying a post processing noise reduction algorithm. Texture information can be visualized using an Inverse Pole Figure (IPF) plot, Figure 2A. The results show that the films have a strong $\langle 111\rangle$ texture perpendicular to the plane of the film, matching our XRD measurements and confirming the validity of these measurements. It is also possible to observe twinning in several of the grains. Crystallographic information can also be used to derive the grain boundary angle between two adjacent grains, Figure 2B. High angle grain boundaries are shown in red while low angle grain boundaries are shown in yellow. CSL boundaries are labelled according to the 
inverse density of their common lattice points.

In summary, EBSD is useful technology for characterizing the crystallographic structure of a variety of samples. However, until recently it has been restricted to low resolution applications due to high electron interaction volume. A dramatic increase in resolution with tEBSD configurations allows us to apply this powerful characterization technique to the nanoscale.

\section{References:}

[1] T. Rausch, A. S. Chu, P. Lu, S. Puranam, D. Nagulapally, and J. Dykes, Recording Performance of a Pulsed HAMR Architecture, (2014).

[2] S. Link, Z. L. Wang, and M. A. El-Sayed, The Journal of Physical Chemistry B 104, 33 (2000).

[3] D. Brandon, Acta Metallurgica 14, 11 (1966).

[4] R. Keller, and R. Geiss, J. Microsc. 245, 3 (2012).

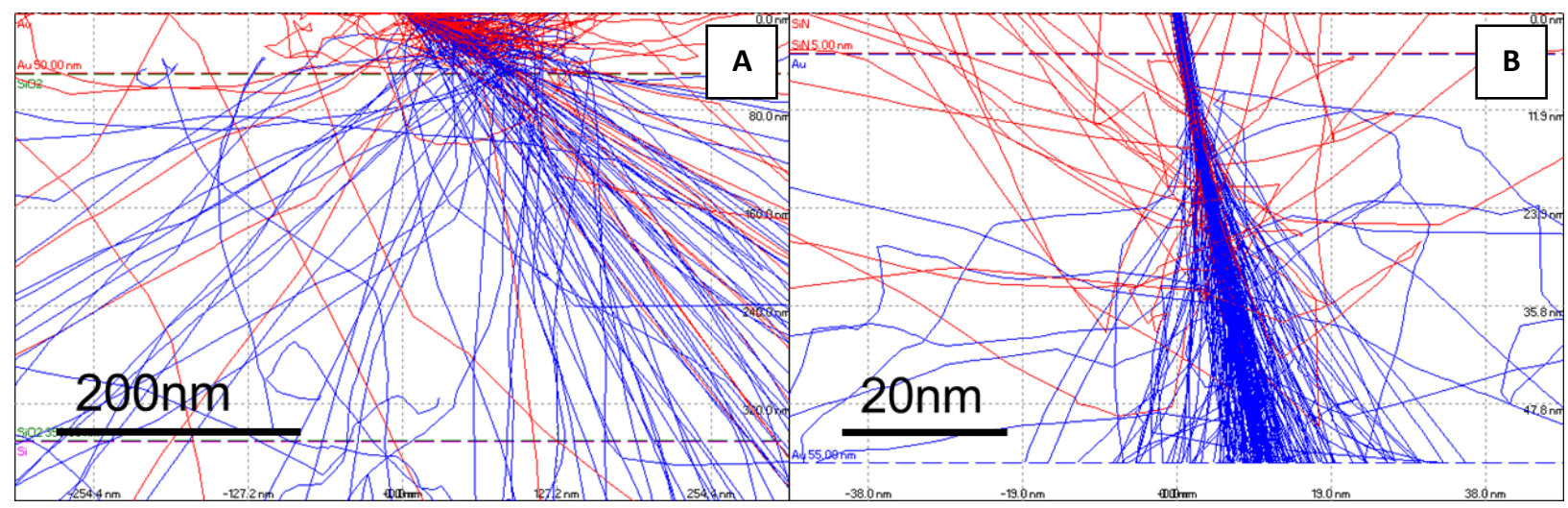

Figure 1. Electron trajectories simulated using CASINO demonstrating the distribution of electrons during conventional (A) and transmission mode (B) EBSD.
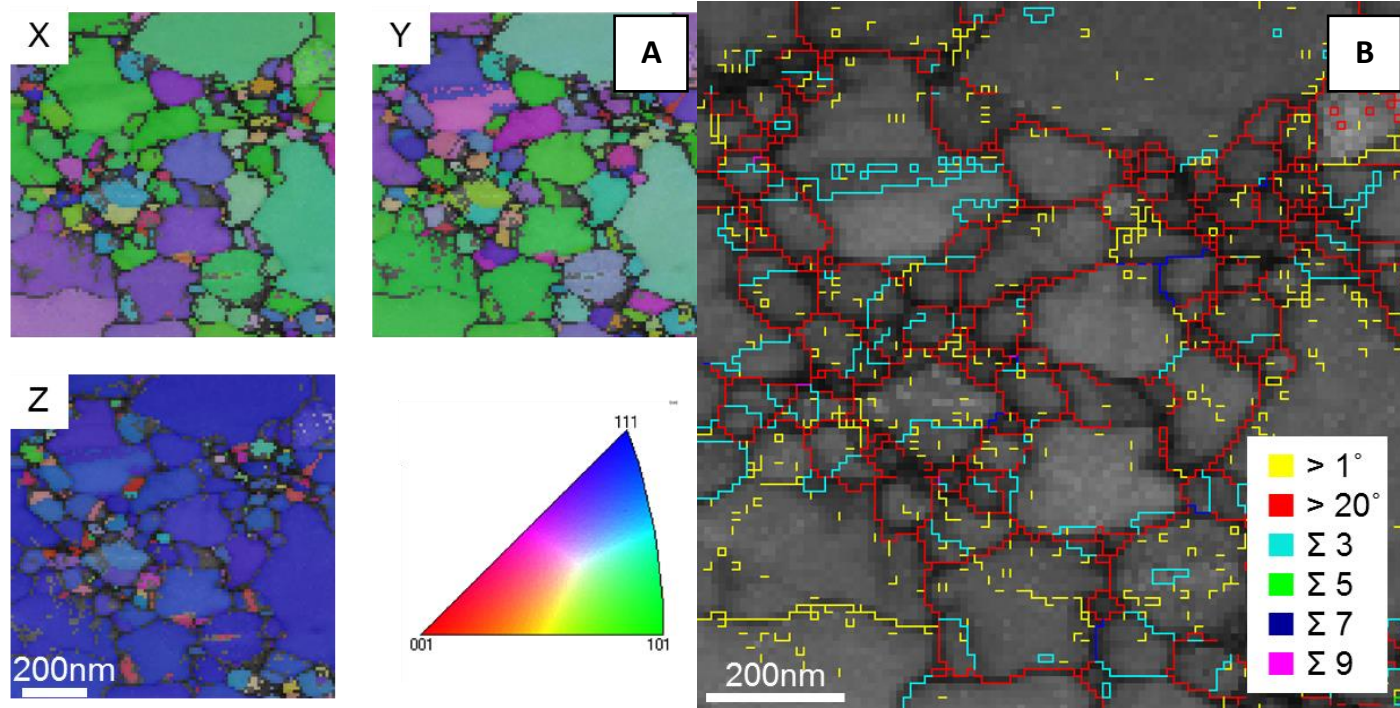

Figure 2. Inverted pole figure diagrams showing film texture in the $\mathrm{X}, \mathrm{Y}$, and $\mathrm{Z}$ (out of plane) directions (A). Grain boundary information showing grain boundary angle and coincidence site lattice boundaries (B). 